\title{
Anderson-Fabry's Disease: \\ A Rare but Treatable Case of Fever of Unknown Origin
}

\author{
Francesca Graziani $^{1}$, Aureliano Ruggio ${ }^{1}$, Antonio laconelli ${ }^{1}$, Elena Verrecchia ${ }^{2}$, Amelia Morrone ${ }^{3}$, \\ Daniela Antuzzi ${ }^{4}$, Filippo $\mathrm{Crea}^{1}{ }^{1}$ Raffaele Manna ${ }^{2}$ \\ ${ }^{1}$ Department of Cardiovascular Medicine, Catholic University of the Sacred Heart, Rome, Italy \\ ${ }^{2}$ Periodic Fever Research Centre, A. Gemelli Policlinic, Catholic University of the Sacred Heart, Rome, Italy \\ ${ }^{3}$ Laboratory for Molecular and Cell Biology of Neurologic and Metabolic Disorders, Paediatric Neurology Unit, Meyer Children's Hospital, Florence, Italy \\ ${ }^{4}$ Department of Paediatric Sciences, A. Gemelli Policlinic, Rome, Italy
}

\section{Doi: 10.12890/2017_000645 - European Journal of Case Reports in Internal Medicine - @ EFIM 2017}

Received: 27/05/2017

Accepted: 07/06/2017

Published: 03/07/2017

How to cite this article: Graziani F, Ruggio A, laconelli A, Verrecchia E, Morrone A, Antuzzi D, Crea F, Manna R. Anderson-Fabry's disease: a rare but treatable case of fever of unknown origin. EJCRIM 2017;4: doi:10.12890/2017_000645.

Conflicts of Interests: The Authors declare that there are no competing interests.

This article is licensed under a Commons Attribution Non-Commercial 4.0 License

\section{ABSTRACT}

Anderson-Fabry's disease (AFD) is a rare, X-linked lysosomal storage disorder caused by the complete deficiency or attenuated activity of the enzyme $\alpha$-galactosidase A, leading to progressive systemic intracellular accumulation of glycosphingolipids and subsequent cellular dysfunction, inflammation and fibrosis. Fever is a frequently misinterpreted symptom in the early stages of the disease, leading to diagnostic delay. We present the case of a 35-year-old man admitted to our Periodic Fever Research Centre for long-lasting recurrent episodes of fever of unknown origin. After extensive assessment, we diagnosed AFD associated with a novel GLA mutation. We started enzyme replacement therapy with clinical benefit and complete remission of fever.

\section{LEARNING POINTS}

- Anderson-Fabry's Disease (AFD) is an inherited lysosomal storage disorder, in which progressive multi-organ glycosphingolipid accumulation leads to multi-systemic dysfunction. Diagnosis requires a high level of suspicion as the clinical presentation can be very heterogeneous.

- As fever is an early uncommon symptom causing diagnostic delay, it is important to consider AFD in the differential diagnosis of recurrent fevers, particularly when febrile episodes are not associated with an increase in acute phase reactants and when other signs or symptoms suggestive of AFD are present.

- Prognosis depends on an early diagnosis because promptly initiation of enzyme replacement therapy (ERT) can prevent the progression of organ damage.In our case fever disappeared after ERT initiation, a finding not previously reported to our knowledge. Therefore, fever remission could be an early marker of response to ERT.

\section{KEYWORDS}

Anderson-Fabry's disease; FUO; Thermoregulation; Enzyme replacement therapy 


\section{CASE DESCRIPTION}

A 35-year-old Italian man was referred to our Periodic Fever Research Centre in October 2013 for fever of unknown origin. He had normal physical and mental development and had received all recommended vaccinations during childhood. He had no history of major diseases and had never travelled in foreign countries. His family history was otherwise unremarkable.

The patient reported he had experienced prolonged recurrent fevers since 5 years of age, lasting 5-10 days on average and with a frequency of five to six episodes per year. Fever was often preceded by prodromal symptoms such as headache, fatigue and asthenia, and was associated with nausea, abdominal pain and sometimes diarrhoea. The patient had been treated with anti-inflammatory drugs and different combinations of wide-spectrum antibiotic drugs, with no clinical benefit.He had also received steroid treatment (prednisone $5 \mathrm{mg} / \mathrm{day}$ ) with transient disappearance of fever, but with prompt relapse on steroid discontinuation.

On July 2013, due to a new, long-lasting episode of such fever, he was admitted to a local hospital, where a complete multi-organ evaluation was performed. Blood count, electrolytes, liver, kidney and thyroid function as well as protein electrophoresis and inflammatory markers (CRP, ESR, B2-microglobulin, fibrinogen, ferritin) were all normal. Screening for autoimmune disorders (including RF, c-ANCA, p-ANCA, ANA, anti-ENA, complement components, anti-thyroglobulin) was negative. Haematological disorders and malignancies were ruled out after bonemarrow biopsy, thoraco-abdominal, head and neck CT scans, and abdominal, prostate and testicular ultrasound. Infectious and parasitic diseases were ruled out by negative serology for CMV, EBV, HIV, toxoplasma, adenovirusand enterovirus, and negative WidalWright and Weil-Felix reactions. Infective endocarditis was excluded by negative blood cultures and transthoracic echocardiography,while central fever was ruled out by brain MRI.

After this extensive screening, the patient was transferred to our hospital for further investigationof the aetiology of his fever. At evaluation, the patient was afebrileand in good general condition, and his physical examination was normal.

During detailed investigation of the symptoms associated with fever, the patient stated that febrile episodes were occasionally associated with acroparesthesias, described as burning and numbness sensations in the extremities with centripetal progression. In addition, in 2007 the patient had experienced acute transient hearing loss and tinnitus in the right ear. A brain MRI performed at that time showed a small right temporal leptomeningeal cyst and dolichoectasia of the vertebrobasilar artery, and the patient was discharged with the diagnosis of 'bilateral perceptive hearing loss for high frequencies'. In 2012 he had an episode of objective vertigo associated with persistent tinnitus in the left ear.

Once we confirmed that the most common causes of fever could be safely excluded, molecular studies for anti-inflammatory fevers (MEFV, TRAPS, MVK) were performed, with negative results. The absence of inflammatory markers during febrile episodes supported this finding. Taken together, recurrent febrile episodes with no increase in acute phase reactants, associated with abdominal pain, a history of acroparesthesias during childhood, the episodes of acute reduction in hearing acuity associated with tinnitus and the incidental finding of dolichoectasia of the vertebrobasilar artery, raised the suspicion of Anderson-Fabry's disease (AFD).

Therefore, $\alpha$-galactosidase activity in peripheral leukocyteswas measured and found to be very low $(4.46 \pm 0.21 \mathrm{nmol} / \mathrm{mg} / \mathrm{h}, \mathrm{normal} \mathrm{range}$ 66.31-88.01). We then performed molecular analysis to confirm the AFD diagnosis and found hemizygosity for a novel GLA gene mutation c.907A>T (p.lle303Phe; data not yet published).

Soon after the diagnosis, extensive assessment of organ involvement was performed. Cardiac evaluation revealed a nearly normal ECG, but the echocardiogram showed mild biventricular hypertrophy.

Other relevant findings were: kidney involvement, as shown by glomerular hyperfiltration (creatinine clearance $166 \mathrm{ml} / \mathrm{min} / 1.73 \mathrm{~m}{ }^{2}, \mathrm{normal}$ range 80-140) with no proteinuria and a normal kidney scan; ear involvement, with bilateral perceptive hearing loss for high frequencies; normal brain MRI except for the dolichoectasia of the vertebrobasilar artery (Fig.1A); and eye involvement, with evidence of bilateral retinal vessel tortuosity (Fig.1B). LysoGb3 levels were $23.6 \mathrm{ng} / \mathrm{ml}$ (normal value $\leq 0.8$ ).

We started enzyme replacement therapy (ERT) and at 6-month follow-up the fever had subsided, paresthesias had improved substantially, and LysoGb3 levels were significantly decreased $(14.1 \mathrm{ng} / \mathrm{ml})$, while left ventricular hypertrophy remained stable.

\section{DISCUSSION}

FD is a rare, X-linked, recessive lysosomal storage disorder caused by complete deficiency or attenuated activity of the enzyme $\alpha$-galactosidase A, leading to progressive systemic intracellular accumulation of neutral glycosphingolipids, particularly globotriaosylceramide, with subsequent cellular dysfunction, inflammation and fibrosis.

In the classic phenotype, progressive glycosphingolipid accumulation in cardiac, renal and cerebrovascular cells leads to multi-organ dysfunction. During childhood, early symptoms are distal extremity pain, recurrent fever, hypo/anhydrosis and gastrointestinal distress. In advanced stages, multi-organ dysfunction causes considerable morbidity and premature death, mainly due to heart and kidney involvement ${ }^{[1,2]}$. 


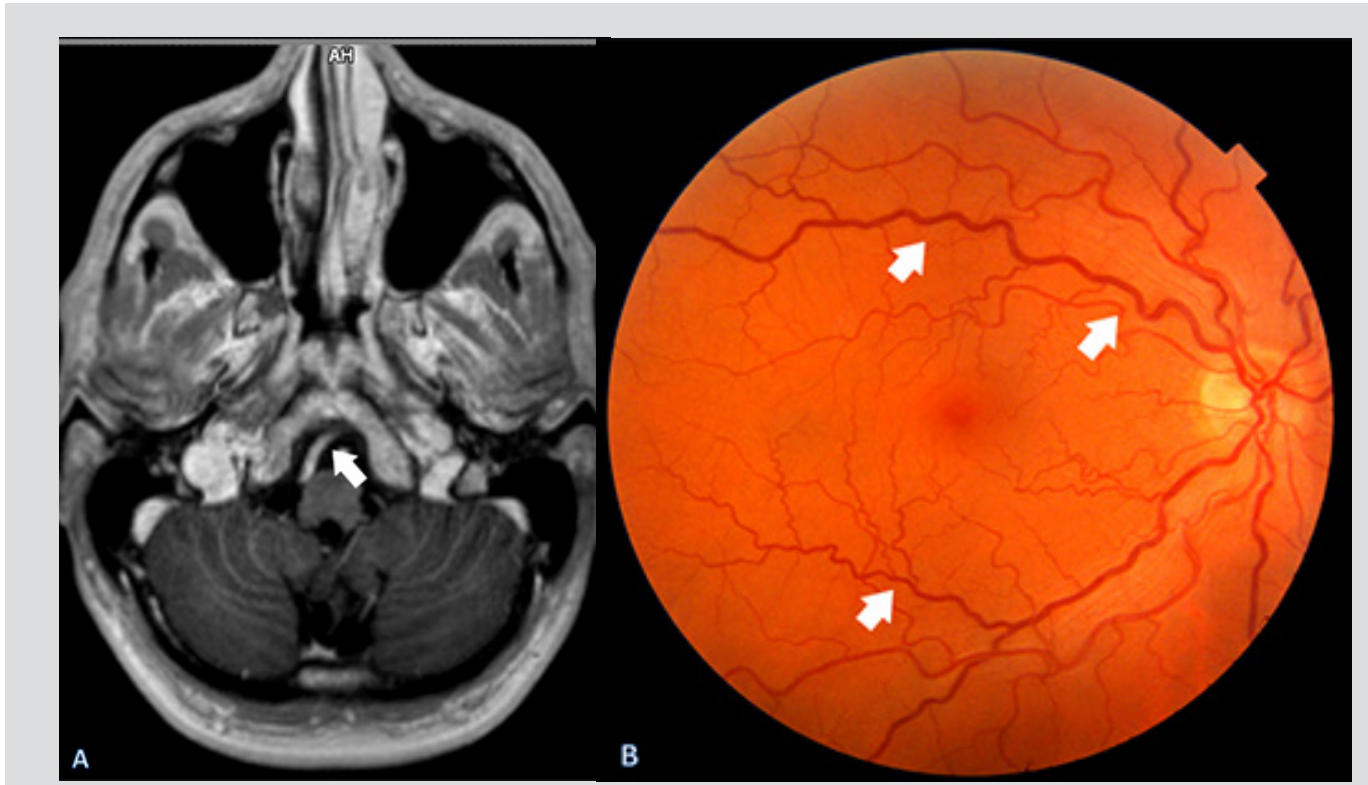

Figure 1 (A) Brain MRI showing dolichoectasia of the vertebrobasilar artery (white arrow), a characteristic finding of Anderson-Fabry's disease. (B) Right ocular fundus showing increased retinal vessels tortuosity with a 'corkscrew' appearance (white arrows).

Fever is a frequently misinterpreted symptom in the early stages of the disease, leading to diagnostic delay. The fever has been attributed to dysfunction of the sweat glands and the cholinergic system, resulting in impaired thermoregulation. A recent retrospective analysis by our group has demonstrated that a significant number of AFD patients presented with fever at the onset of the disease. In our analysis, fever was associated with a significant mean diagnostic delay of $15.8 \pm 12.8$ years ${ }^{[3]}$.

In this case the diagnostic delay was 29 years, which highlights the importance of considering AFD in the differential diagnosis of recurrent fever, particularly when febrile episodes are not associated with an increase in acute phase reactants and when other signs or symptoms suggestive of AFD are present.

Prompt diagnosis allows the administration of specific therapy to modify the course of the disease. Early initiation of ERT can prevent progression to organ damage, stabilize the existing disease and prevent or delay major complications ${ }^{[4]}$.

\section{REFERENCES}

1. Zarate YA, Hopkin RJ. Fabry's disease. Lancet 2008;372:1427-1435.

2. Weidemann F, Linhart A, Monserrat L, Strotmann J. Cardiac challenges in patients with Fabry disease. Int J Cardiol 2010:141:3-10.

3. Verrecchia E, Zampetti A, Antuzzi D, Ricci R, Ferri L, Morrone A et al. The impact of fever/hyperthermia in the diagnosis of Fabry: a retrospective analysis. Eur J Intern Med 2016;32:26-30.

4. Weidemann F, Niemann M, Breunig F, Herrmann S, Beer M, Störk S et al. Long-term effects of enzyme replacement therapy on Fabry cardiomyopathy: evidence for a better outcome with early treatment. Circulation 2009;119:524-529. 